\title{
Evaluation of Seal Effects on the Stability of Rotating Fluid Machinery
}

\author{
T. IWATSUBO \\ Faculty of Engineering, Kobe University, 1-1 Rokkodai-cho Nada-ku, Kobe, Japan \\ B. C. SHENG \\ KUBOTA Corporation, 1-2-27 Shiromi Chuo-ku Osaka City, Japan
}

\begin{abstract}
The stability of typical rotating fluid machinery such as single and multi-stage pumps is evaluated by using the finite element method. The individual contribution of the impellers, bearings and seals to the stability and the dynamic interactions of these fluid elements are examined. Various types of bearings and seals, such as annular smooth, parallel grooved and damper seals, are compared for better rotor stability. The effect of the operating conditions on the stability is also investigated. The results show that rotor stability can be easily improved by replacing the unstable fluid elements.
\end{abstract}

Key Words: Rotating fluid machinery; Bearing; Impeller; Non-contacting seal; Rotor stability; Logarithmic decrement

\section{INTRODUCTION}

I $\mathrm{n}$ recent years, the operating conditions of rotating fluid machinery tend towards higher speed and higher pressure along with the rapid progress made in the technology in industry and space development. In order to raise the efficiency and prevent leakage flow at high pressure, the clearance of fluid elements such as bearings, noncontacting seals, and impellers has to be designed possibly smaller. However, with higher pressure, higher speed, and smaller clearance, greater fluid forces occur and sometimes these forces cause unstable vibration.

To prevent such unstable vibration, firstly the dynamic characteristics of these fluid elements and their individual contribution to rotor stability must be made clear. Work is being done, and many results have been obtained by various researchers, [1], [2], [4]-[8], [10], [11]. Secondly, the combined effect of these fluid elements on rotor stability and their interaction must be investigated. Yang et al. [1985] investigated the effect of annular smooth and taper seals on the stability of the single-stage pump rotor system. Diewald et al. [1987] showed a procedure to investigate the effect of annular smooth and grooved seals and impellers on the stability of the Jeffcott rotor and the multi-stage pump rotor system. These researches show that the rotor stability is strongly affected by the fluid elements, and the contribution of these elements to rotor stability varies according to the operating conditions.

In this paper, continuing the research of Yang et al. [1985], the stability of typical rotating fluid machinery such as the single-stage and multi-stage pumps, which consist of impellers, bearings, and non-contacting seals, are evaluated by using the finite element method. The individual contribution of the impellers, bearings as well as seals to the stability, and the dynamic interactions of these fluid elements are investigated. The contribution to the rotor stability is evaluated by the logarithmic decrement. For the linear and non-cross-coupled inertia rotor system, the total logarithmic decrement of the rotor system can be represented as the sum of the individual decrements. Therefore, the stability of the rotor system can be easily improved by changing the unstable fluid elements in the design stage. In the investigation, some types of bearings and seals such as annular smooth, parallel grooved and damper seals are compared to seek better ones for the 
rotor stability. The effect of the operating conditions on stability is also studied.

\section{EQUATIONS OF MOTION AND LOGARITHMIC DECREMENT}

The analytical models of the single and multi-stage pump are shown in Figures 1 and 2. For general application, the non-symmetrical single-stage pump is taken. These rotor systems consist of bearings, impellers and seals. For the convenience of analysis, the impeller is looked upon as a disk which has the same mass and same moment of inertia as the practical impeller. If the rotor rotates with a steady angular velocity $\omega$, the equation of motion of the disk elements in the coordinates illustrated in Figure 2 is as follows:

$$
\left(\left[M_{t}^{d}\right]+\left[M_{r}^{d}\right]\right)\left\{\ddot{q}^{d}\right\}-\omega\left[G^{d}\right]\left\{\dot{q}^{d}\right\}=\left\{F^{d}\right\}
$$

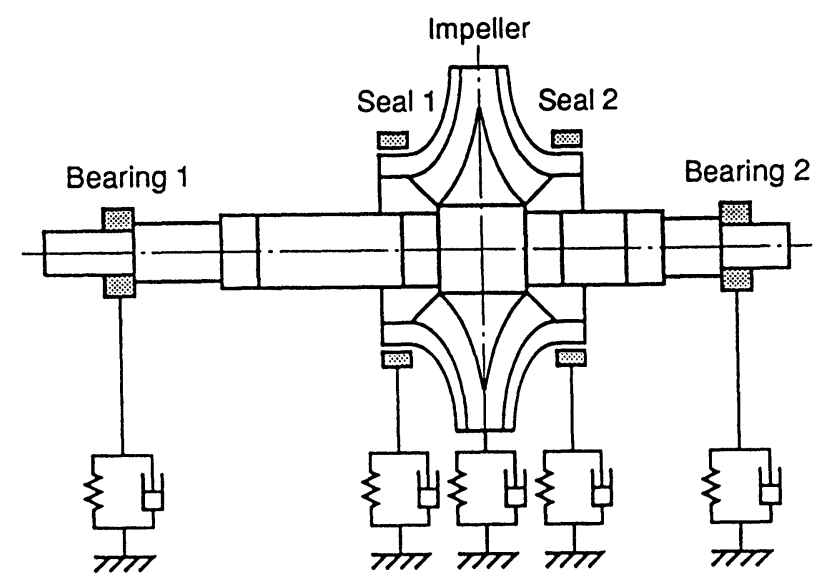

FIGURE 1 Model of single-stage pump. where $\left[M_{t}^{d}\right]$ and $\left[M_{r}^{d}\right]$ are respectively the mass matrices for translational and bending motions; $\left[G^{d}\right]$ is gyroscopic matrix; $\left\{F^{d}\right\}$ is the force vector acting on the disks.

The equation of motion of the journal elements is given by the expression

$$
\left(\left[M_{t}^{e}\right]+\left[M_{r}^{e}\right]\right)\left\{\ddot{q}^{e}\right\}-\omega\left[G^{e}\right]\left\{\dot{q}^{e}\right\}+\left[K^{e}\right]\left\{q^{e}\right\}=\left\{F^{e}\right\}
$$

where $\left\{q^{e}\right\}=\left\{q_{t}, q_{r}\right\}^{T}=\left\{x, y, \phi_{x}, \phi_{y}\right\}^{T},\left[M_{t}^{e}\right],\left[M_{r}^{e}\right]$, $\left[G^{e}\right],\left[K^{e}\right]$ are the mass matrices of translational and bending motions, gyroscopic matrix and stiffness matrix, respectively; $\left\{F^{e}\right\}$ is the fluid force vector of bearings, impellers and seals, and they are expressed as follows:

$$
\begin{aligned}
-\left\{F_{B}\right\} & =\left[C_{B}\right]\left\{\dot{q}_{t}\right\}+\left[K_{B}\right]\left\{q_{t}\right\} \\
-\left\{F_{I}\right\} & =\left[M_{I}\right]\left\{\ddot{q}_{t}\right\}+\left[C_{I}\right]\left\{\dot{q}_{t}\right\}+\left[K_{I}\right]\left\{q_{t}\right\} \\
-\left\{F_{S}\right\} & =\left[M_{S}\right]\left\{\ddot{q}_{t}\right\}+\left[C_{S}\right]\left\{\dot{q}_{t}\right\}+\left[K_{S}\right]\left\{q_{t}\right\}
\end{aligned}
$$

where $\left[M_{I}\right],\left[M_{S}\right]$ are the inertia coefficient matrices; $\left[C_{B}\right],\left[C_{I}\right],\left[C_{S}\right]$ are the damping coefficient matrices; $\left[K_{B}\right],\left[K_{I}\right],\left[K_{S}\right]$ are the stiffness coefficient matrices. Substituting eq. (3) into eq. (2), and combining the equations of disk elements and journal elements, the equation motion of the rotor system can be obtained.

$$
[M]\{\ddot{q}\}+[C]\{\dot{q}\}+[K]\{q\}=\{F\}
$$

where $\{F\}$ is the external force not including the fluid forces of bearings, impellers and seals. To determine the eigenvalues and eigenvectors, the characteristic equation

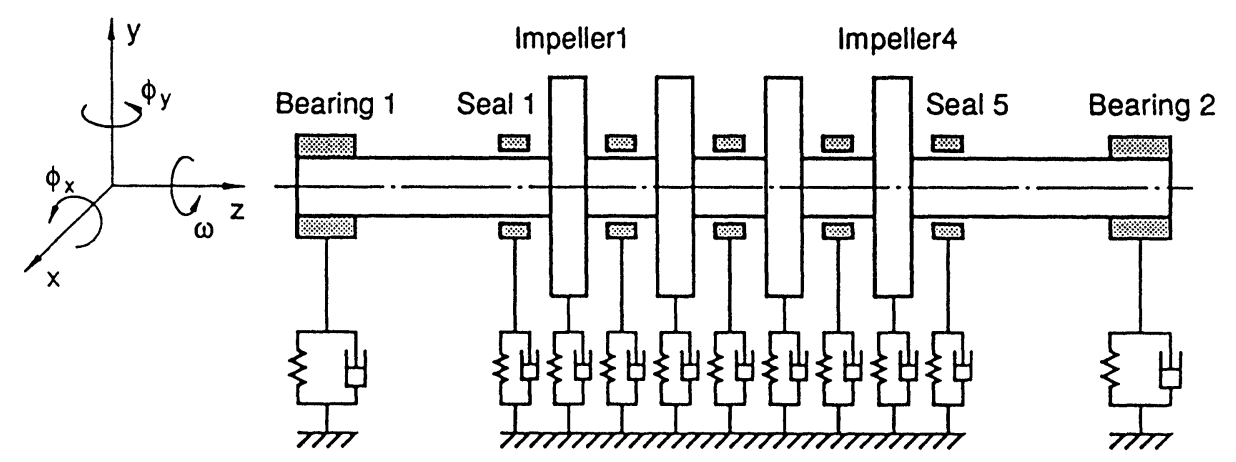

FIGURE 2 Model of multi-stage pump. 
of eq. (4) is rewritten in the following form:

$$
\left[\begin{array}{cc}
{[I]} & 0 \\
{[C]} & {[M]}
\end{array}\right]\left\{\begin{array}{l}
\dot{q} \\
\ddot{q}
\end{array}\right\}+\left[\begin{array}{cc}
0 & -[I] \\
{[K]} & 0
\end{array}\right]\left\{\begin{array}{l}
q \\
\dot{q}
\end{array}\right\}=\left\{\begin{array}{l}
0 \\
0
\end{array}\right\}
$$

or

$$
[D]\{\dot{z}\}+[E]\{z\}=\{0\}
$$

Letting the generalized solution of eq. (6) have the form

$$
\{z\}=\{\bar{z}\} e^{\lambda t}
$$

then eq. (6) yields

$$
\left\{[I] \lambda+[D]^{-1}[E]\right\}\{\bar{q}\}=\{0\}
$$

where $\lambda$ and $\{\bar{q}\}$ are the eigenvalue and eigenvector, respectively. They can be obtained by solving eq. (8).

In general, the eigenvalues are conjugate complex, and expressed in the following form:

$$
\lambda_{i}=\alpha_{i}+j \omega_{i}, \quad \bar{\lambda}_{i}=\alpha_{i}-j \omega_{i}
$$

where $i$ means $i$ th natural mode. The logarithmic decrement of the rotor system is defined as

$$
\delta_{i}=-2 \pi \frac{\alpha_{i}}{\omega_{i}}
$$

In order to investigate the effects of the fluid elements on the rotor stability, the logarithmic decrements of the fluid elements have to be determined. These logarithmic decrements can be obtained by the eigenvalue and eigenvector through the following transformation (Kurohashi et al. [1982].

Substituting the $i$ th conjugate eigenvalues $\lambda_{i}, \bar{\lambda}_{i}$ and $i$ th conjugate eigenvectors $\left\{\psi_{i}\right\},\left\{\bar{\psi}_{i}\right\}$ into the characteristic equation of eq. (4), the following equations are obtained.

$$
\begin{aligned}
& \lambda_{i}^{2}[M]\left\{\psi_{i}\right\}+\lambda_{i}[C]\left\{\psi_{i}\right\}+[K]\left\{\psi_{i}\right\}=\{0\} \\
& \bar{\lambda}_{i}^{2}[M]\left\{\bar{\psi}_{i}\right\}+\bar{\lambda}_{i}[C]\left\{\bar{\psi}_{i}\right\}+[K]\left\{\bar{\psi}_{i}\right\}=\{0\}
\end{aligned}
$$

Furthermore, expressing the mass matrix, damping matrix and stiffness matrix as the sum of symmetric parts $M^{*}, C^{*}, K^{*}$ and unsymmetric parts $\Delta M, \Delta C, \Delta K$, then the above equations become

$$
\begin{aligned}
& \lambda_{i}^{2}\left[M^{*}+\Delta M\right]\left\{\psi_{i}\right\}+\lambda_{i}\left[C^{*}+\Delta C\right]\left\{\psi_{i}\right\} \\
& +\left[K^{*}+\Delta K\right]\left\{\psi_{i}\right\}=\{0\} \\
& \bar{\lambda}_{i}^{2}\left[M^{*}+\Delta M\right]\left\{\bar{\psi}_{i}\right\}+\bar{\lambda}_{i}\left[C^{*}+\Delta C\right]\left\{\bar{\psi}_{i}\right\} \\
& \quad+\left[K^{*}+\Delta K\right]\left\{\bar{\psi}_{i}\right\}=\{0\}
\end{aligned}
$$

where $[\Delta M]=-[\Delta M]^{T},[\Delta C]=-[\Delta C]^{T},[\Delta K]=$ $-[\Delta K]^{T}$.
Premultiplying equations of (12) by $\left\{\bar{\psi}_{i}\right\}^{T}$ and $\left\{\psi_{i}\right\}^{T}$, and introducing the following expressions

$$
\begin{aligned}
\left\{\bar{\psi}_{i}\right\}^{T}\left[M^{*}\right]\left\{\psi_{i}\right\} & =\left\{\psi_{i}\right\}^{T}\left[M^{*}\right]\left\{\bar{\psi}_{i}\right\}=m_{i}^{*} \\
\left\{\bar{\psi}_{i}\right\}^{T}\left[C^{*}\right]\left\{\psi_{i}\right\} & =\left\{\psi_{i}\right\}^{T}\left[C^{*}\right]\left\{\bar{\psi}_{i}\right\}=c_{i}^{*} \\
\left\{\bar{\psi}_{i}\right\}^{T}\left[K^{*}\right]\left\{\psi_{i}\right\} & =\left\{\psi_{i}\right\}^{T}\left[K^{*}\right]\left\{\bar{\psi}_{i}\right\}=k_{i}^{*} \\
\left\{\bar{\psi}_{i}\right\}^{T}[\Delta M]\left\{\psi_{i}\right\} & =-\left\{\psi_{i}\right\}^{T}[\Delta M]\left\{\bar{\psi}_{i}\right\}=j \Delta m_{i} \\
\left\{\bar{\psi}_{i}\right\}^{T}[\Delta C]\left\{\psi_{i}\right\} & =-\left\{\psi_{i}\right\}^{T}[\Delta C]\left\{\bar{\psi}_{i}\right\}=j \Delta c_{i} \\
\left\{\bar{\psi}_{i}\right\}^{T}[\Delta K]\left\{\psi_{i}\right\} & =-\left\{\psi_{i}\right\}^{T}[\Delta K]\left\{\bar{\psi}_{i}\right\}=j \Delta k_{i}
\end{aligned}
$$

the difference of the two equations of eq. (12) can be written as follows:

$$
\begin{gathered}
\left(\lambda_{i}^{2}-\bar{\lambda}_{i}^{2}\right) m_{i}^{*}+\left(\lambda_{i}^{2}+\bar{\lambda}_{i}^{2}\right) \Delta m_{i}+\left(\lambda_{i}+\bar{\lambda}_{i}\right) c_{i}^{*} \\
-\left(\lambda_{i}+\bar{\lambda}_{i}\right) \Delta c_{i}+2 \Delta k_{i}=0
\end{gathered}
$$

Because the unsymmetric mass $\Delta m_{i}$ is caused by the impellers and seals, and it is usually much smaller than the symmetric mass $m_{i}^{*}$, it is ignored here. Substituting eq. (9) into eq. (14), the real part of the eigenvalues is obtained.

$$
\alpha_{i}=-\frac{\omega_{i} c_{i}^{*}+\Delta k_{i}}{2 \omega_{i} m_{i}^{*}+\Delta c_{i}}
$$

Therefore, the logarithmic decrement is expressed as follows:

$$
\delta_{i}=-2 \pi \frac{\alpha_{i}}{\omega_{i}}=\frac{\pi \omega_{i} c_{i}^{*}+\pi \Delta k}{\omega_{i}^{2} m_{i}^{*}+\omega_{i} \Delta c_{i} / 2}
$$

For the present rotor systems, $c_{i}^{*}$ and $\Delta k_{i}$ can be expressed as the sum of the fluid elements.

$$
\begin{aligned}
c_{i}^{*}= & \left\{\bar{\psi}_{i}\right\}^{T}\left[C^{*}\right]\left\{\psi_{i}\right\} \\
= & \sum_{j=1}^{n_{B}}\left(\left\{\bar{\psi}_{B i}\right\}_{j}^{T}\left[C_{B}^{*}\right]_{j}\left\{\bar{\psi}_{B i}\right\}_{j}\right) \\
& +\sum_{j=1}^{n_{S}}\left(\left\{\bar{\psi}_{S i}\right\}_{j}^{T}\left[C_{S}^{*}\right]_{j}\left\{\psi_{S i}\right\}_{j}\right) \\
& +\sum_{j=1}^{n_{I}}\left(\left\{\bar{\psi}_{I i}\right\}_{j}^{T}\left[C_{I}^{*}\right]_{j}\left\{\psi_{I i}\right\}_{j}\right) \\
= & \sum_{j=1}^{n_{B}}\left(c_{B i}^{*}\right)_{j}+\sum_{j=1}^{n_{S}}\left(c_{S i}^{*}\right)_{j}+\sum_{j=1}^{n_{I}}\left(c_{I i}^{*}\right)_{j}
\end{aligned}
$$




$$
\begin{aligned}
\Delta k_{i}= & \left\{\bar{\psi}_{i}\right\}^{T}[\Delta k]_{j}\left\{\psi_{i}\right\} \\
= & \sum_{j=1}^{n_{B}}\left(\left\{\bar{\psi}_{B i}\right\}_{j}^{T}\left[\Delta k_{B}\right]_{j}\left\{\psi_{B i}\right\}_{j}\right) \\
& +\sum_{j=1}^{n_{S}}\left(\left\{\bar{\psi}_{S i}\right\}_{j}^{T}\left[\Delta k_{S}\right]_{j}\left\{\psi_{S i}\right\}_{j}\right) \\
& +\sum_{j=1}^{n_{I}}\left(\left\{\bar{\psi}_{I i}\right\}_{j}^{T}\left[\Delta k_{I}\right]_{j}\left\{\psi_{I i}\right\}_{j}\right) \\
= & \sum_{j=1}^{n_{B}}\left(\Delta k_{B i}\right)_{j}+\sum_{j=1}^{n_{S}}\left(\Delta k_{S i}\right)_{j}+\sum_{j=1}^{n_{I}}\left(\Delta k_{I i}\right)_{j}
\end{aligned}
$$

Substituting the above expressions into eq. (16), the logarithmic decrement expressed as the sum of the individual elements is obtained.

$$
\begin{aligned}
\delta_{i}= & \frac{\pi}{\omega_{i}^{2} m_{i}^{*}+\omega_{i} \Delta c_{i} / 2} \\
& \times\left\{\omega_{i}\left[\sum_{j=1}^{n_{B}}\left(c_{B i}\right)_{j}+\sum_{j=1}^{n_{S}}\left(c_{S i}\right)_{j}+\sum_{j=1}^{n_{I}}\left(c_{I i}\right)_{j}\right]\right. \\
& \left.+\left[\sum_{j=1}^{n_{B}}\left(\Delta k_{B i}\right)_{j}+\sum_{j=1}^{n_{S}}\left(\Delta k_{S i}\right)_{j}+\sum_{j=1}^{n_{I}}\left(\Delta k_{I i}\right)_{j}\right]\right\} \\
= & \sum_{j=1}^{n_{B}} \delta_{B i j}+\sum_{j=1}^{n_{S}} \delta_{S i j}+\sum_{j=1}^{n_{I}} \delta_{I i j}
\end{aligned}
$$

where $n_{B}, n_{S}$, and $n_{I}$ are the total numbers of the bearings, seals and impellers, respectively.

\section{EXAMPLE OF ANALYSIS}

\section{Stability of Single-Stage Pumps}

The calculation is based on the conditions in Table I. In the calculation, the bearing dynamic coefficients and impeller dynamic coefficients of JSME [1984] and Ohashi and Shoji [1987] are used, while the dynamic coefficients of seals are obtained by the calculating method given in Iwatsubo and Sheng [1989]; [1990].

The loci of eigenvalues of eq. (8) for single-stage pump rotor systems with smooth, damper and parallel grooved seals are illustrated in Figure 3. Here, only the two eigenvalues in or near to the unstable region $\left(\alpha_{i} \geq 0\right)$ are given. In these figures, $\omega_{0}$ is the first eigenfrequency of the rotor without seals and bearings. The numbers marked on

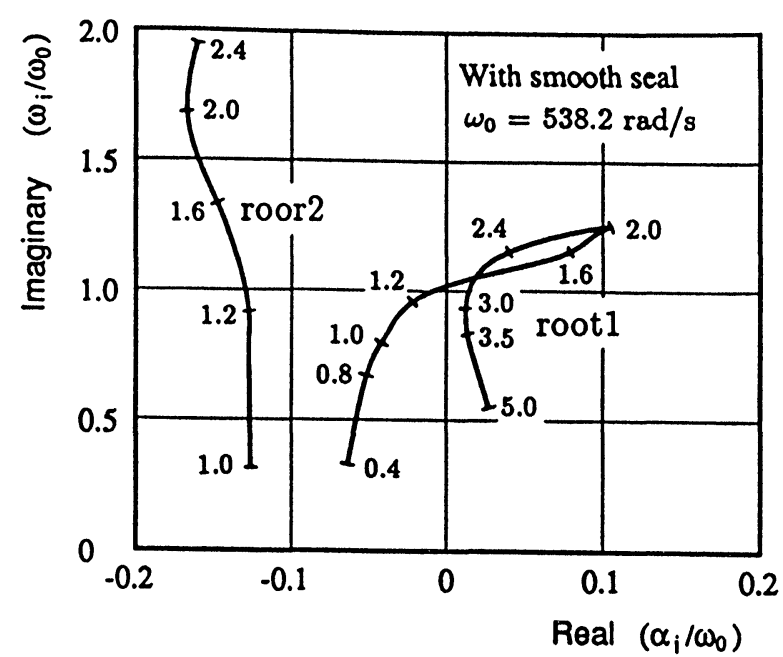

(a) With smooth seals

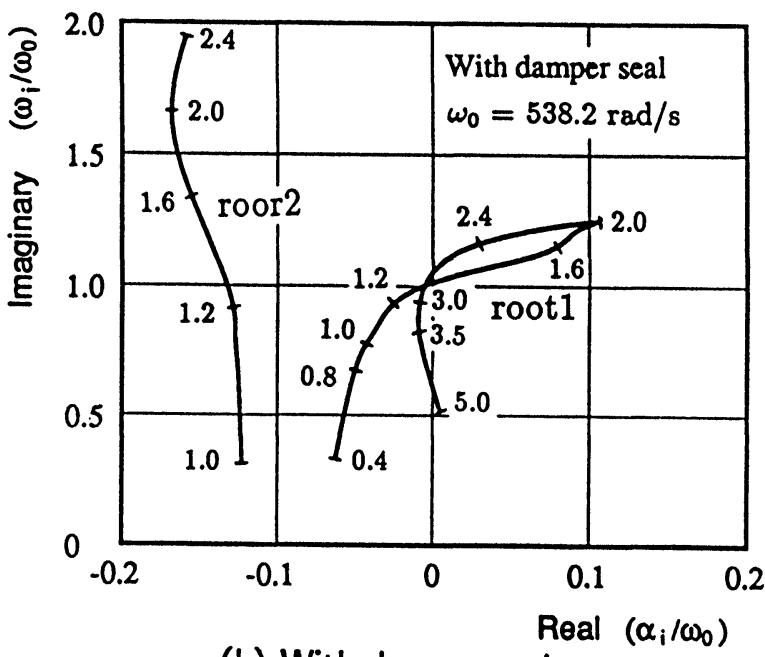

(b) With damper seals

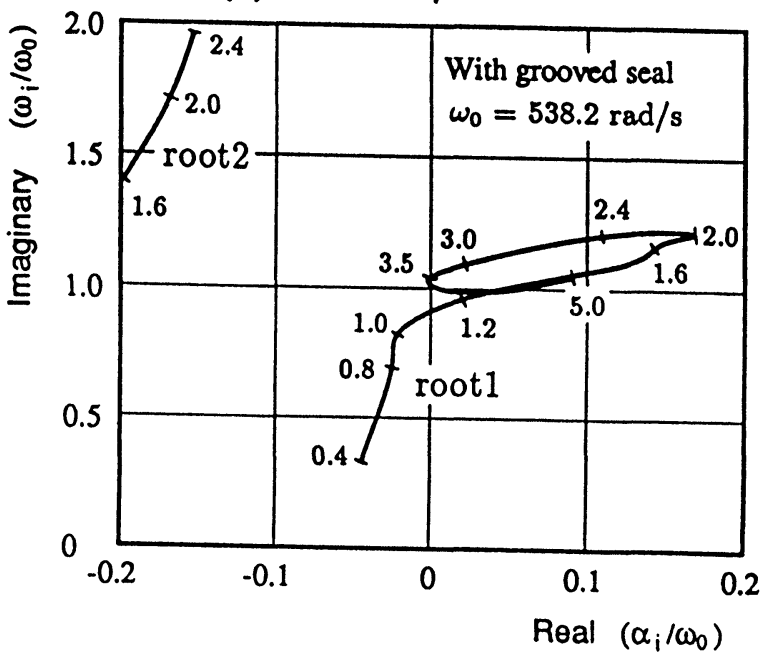

FIGURE 3 Complex eigenvalues. 


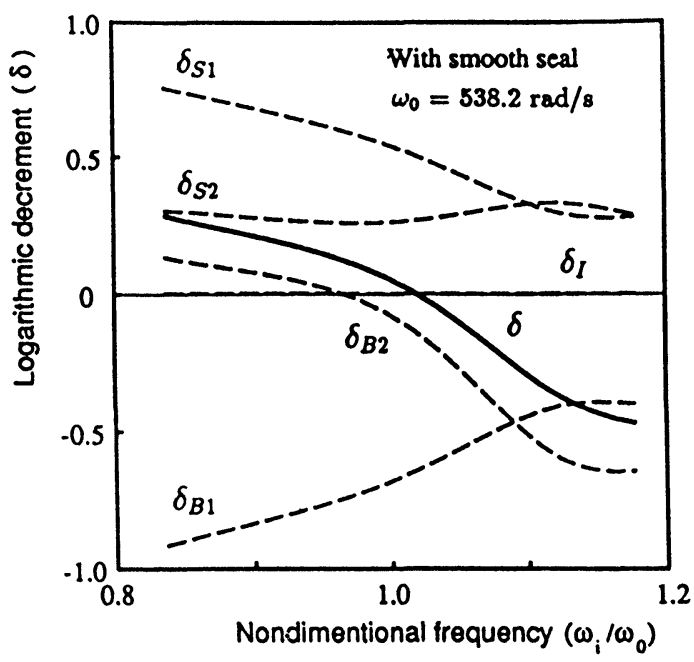

(a) With smooth seals

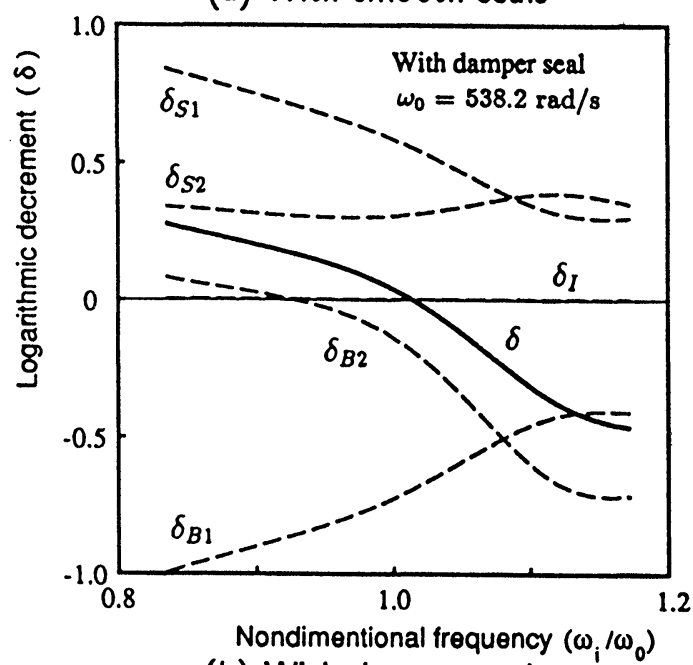

(b) With damper seals

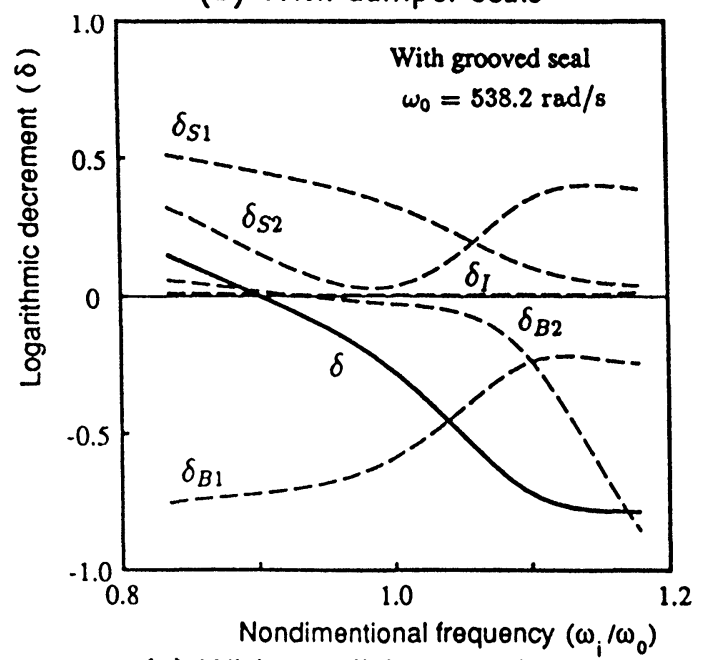

(c) With parallel grooved seals

FIGURE 4 Logarithmic decrements.
TABLE I

Specification of Single-Stage Pump

\begin{tabular}{llc}
\hline Bearing length & $(\mathrm{mm})$ & 60 \\
Bearing diameter & $(\mathrm{mm})$ & 60 \\
Bearing clearance & $(\mathrm{mm})$ & 0.06 \\
Seal length & $(\mathrm{mm})$ & $20-40$ \\
Seal diameter & $(\mathrm{mm})$ & 120 \\
Seal clearance & $(\mathrm{mm})$ & $0.2-0.6$ \\
Impeller width & $(\mathrm{mm})$ & 80 \\
Impeller diameter & $(\mathrm{mm})$ & 300 \\
Impeller clearnace & $(\mathrm{mm})$ & 0.5 \\
Pressure difference & $(\mathrm{MPa})$ & 4.9 \\
Oil viscosity & $(\mathrm{mPa} \cdot \mathrm{s})$ & 16.7 \\
Oil temperature & $\left({ }^{\circ} \mathrm{C}\right)$ & 60 \\
Water temperature & $\left({ }^{\circ} \mathrm{C}\right)$ & 50 \\
\hline
\end{tabular}

the loci are the ratios at rotating speed $N$ to $\omega_{0}$. In the present rotating region, the stability of the rotor system is dependent on the eigenvalue of root 1 . Therefore, the stability is discussed as to this eigenvalue by means of the logarithmic decrement.

From the point of view of energy, the rotor system is releasing energy to the outside so that the system tends to stabilize with the lapse of time, if the logarithmic decrement is positive; while, if the logarithmic decrement is negative, the rotor system is absorbing energy from the outside so that the system enlarges the amplitude and tends to unstablize with the lapse of time (Kurohashi et al. [1982]). Figure 4 shows the logarithmic decrements of the bearings, seals, impellers and the total rotor systems. For this natural mode, the contribution of the impeller to the stability is very small. The logarithmic decrements of the seals are positive, which means the stable effect on the rotor stability, but the logarithmic decrements of the bearings are almost negative, which means an unstable effect on the rotor stability.

The logarithmic decrements of the rotor systems with smooth, damper and parallel grooved seals are shown in Figure 5. The results show that the logarithmic decrements of rotor systems with smooth and damper seals are similar, and the stability of these systems are better than that of rotor systems with parallel grooved seals. In actual machinery, investigation of rotor stability with a specific rotating speeds or in a specific rotating region is usually necessary. The logarithmic decrements of rotor systems with different seals at specific rotating speed $N / \omega_{0}=1$ are shown in Figure 6, where the mode shape of the rotor system is shown, too. These results are drawn in the form of bar graphs for the convenience of clarity. From these results the contribution of every fluid element to rotor stability can be immediately recognized. The rotor stability can be improved by replacing the unstable elements with stable elements. This will be discussed in next section. 


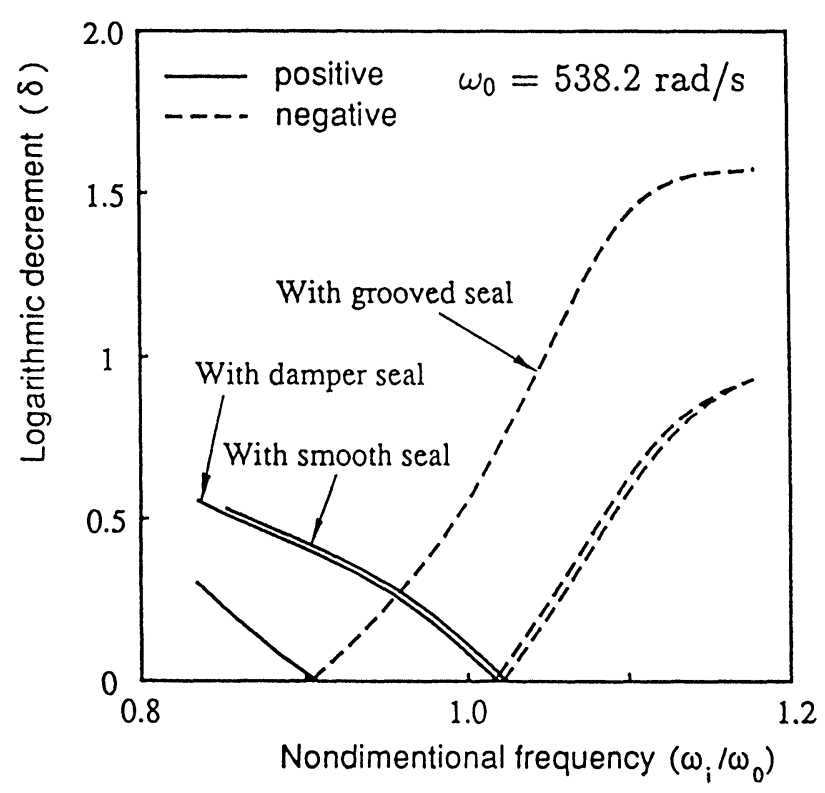

FIGURE 5 Comparison of logarithmic decrement.

The influence of preswirl velocity $V_{t}$ in seals on rotor stability is investigated, and the results are shown in Figure 7. This figure shows that positive preswirl velocity exerts an unstable influence on rotor stability; while neg- ative preswirl velocity exerts a stable influence on rotor stability. This result is in agreement with the individual research of seals (see Iwatsubo et al. [1989]; Iwatsubo and Sheng [1989]; [1990]).

\section{Stability of Multi-stage Pumps}

The specification of multi-stage pumps is illustrated in Table II. The logarithmic decrements of rotor systems and fluid elements at $N=\omega_{0}=19671.6 \mathrm{rpm}$ are studied and shown in Figure 8, where the mode shapes of rotor systems are shown, too. In the investigation, some of seals or bearings are changed in order to improve rotor stability and find the interactions of these fluid elements.

In the case of (a), five parallel grooved seals are used in the rotor system. The total logarithmic decrement shows a negative value because of the unstable effects of the seals and the bearings. If the parallel grooved seal 1 is replaced by a damper seal, the rotor system becomes stable (b). Furthermore, by replacing all the grooved seals with damper seals, the rotor system becomes more stable (c). It is found that after the replacement of the seals, not only the logarithmic decrements of the seals but also those of the bearings are changed. If the circle bearings are replaced with tilting pad bearings instead of the seals, as

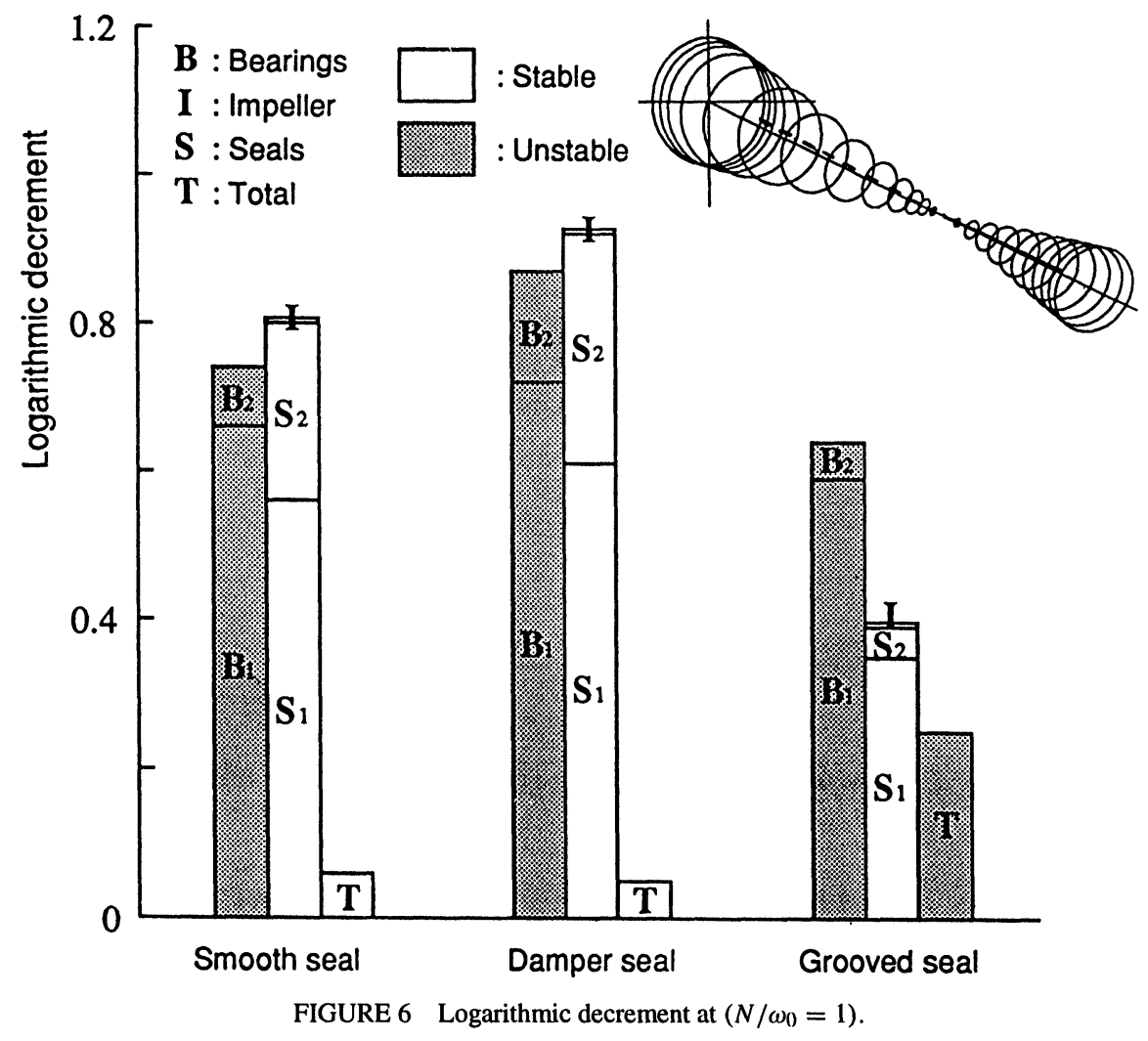




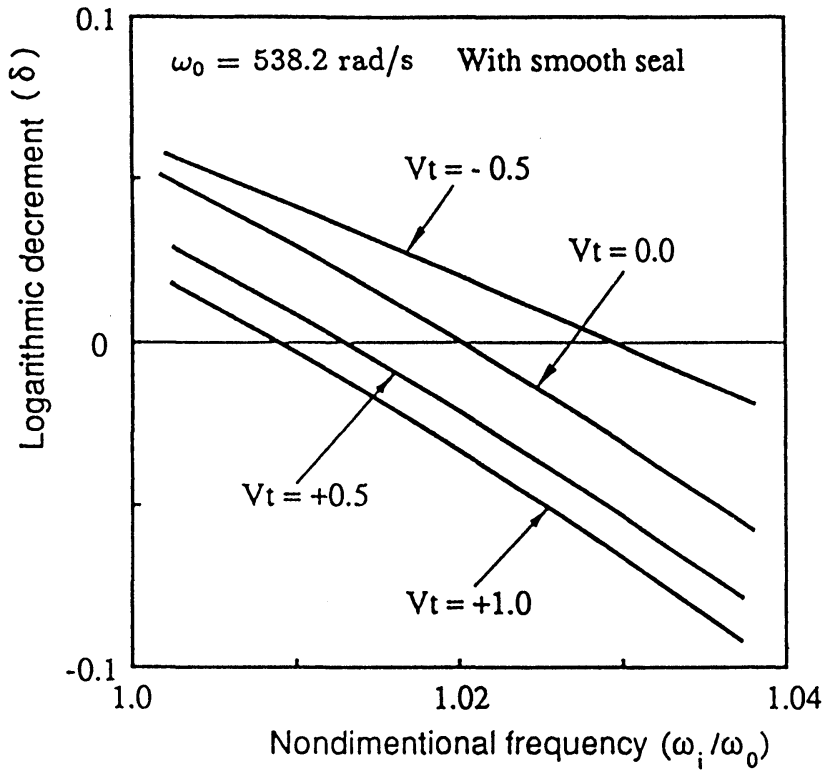

FIGURE 7 Effect of preswirl velocity on logarithmic decrement.

shown in (d), the rotor stability can also be improved. In this case, the logarithmic decrements of the seals are also changed. It is considered that the interactive variation of the fluid elements is caused by the variation of the natural mode, because the logarithmic decrement is dependent on the natural mode, which has been demonstrated in the previous section. According to the above discussion, the interaction of other elements must be considered when evaluating the effect of the fluid element on rotor stability.

\section{CONCLUSION}

The present analysis supports the following conclusions:

1. In rotating fluid machinery, the investigation of the

TABLE II

Specification of Multi-Stage Pump

\begin{tabular}{llc}
\hline Bearing length & $(\mathrm{mm})$ & 80 \\
Bearing diameter & $(\mathrm{mm})$ & 60 \\
Bearing clearance & $(\mathrm{mm})$ & 0.06 \\
Seal length & $(\mathrm{mm})$ & $20-40$ \\
Seal diameter & $(\mathrm{mm})$ & 100 \\
Seal clearance & $(\mathrm{mm})$ & $0.2-0.6$ \\
Impeller width & $(\mathrm{mm})$ & 40 \\
Impeller diameter & $(\mathrm{mm})$ & 300 \\
Impeller clearance & $(\mathrm{mm})$ & 0.5 \\
Pressure difference & $(\mathrm{MPa})$ & 4.9 \\
Oil viscosity & $(\mathrm{mPa} \cdot \mathrm{s})$ & 16.7 \\
Oil temperature & $\left({ }^{\circ} \mathrm{C}\right)$ & 60 \\
Water temperature & $\left({ }^{\circ} \mathrm{C}\right)$ & 50 \\
\hline
\end{tabular}

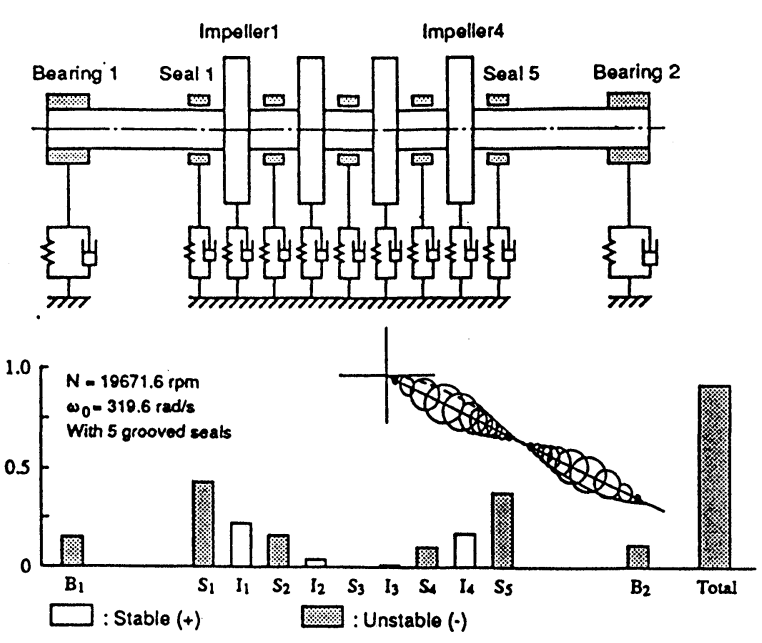

(a) With 2 circle journal bearings and 5 grooved seals

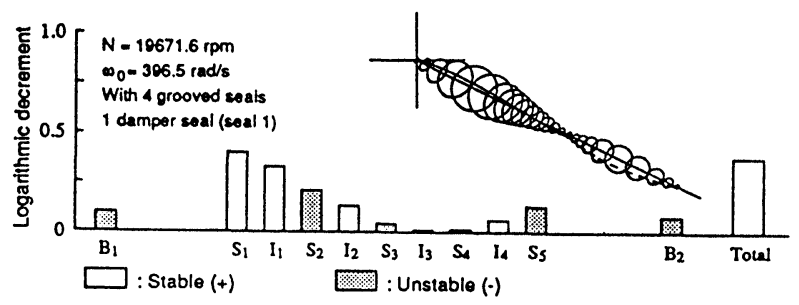

(b) With 2 circle journal bearings,

4 grooved seals and 1 damper seal

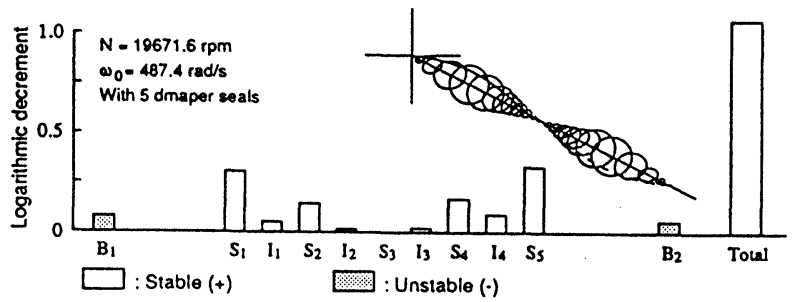

(c) With 2 circle journal bearings and 5 damper seals

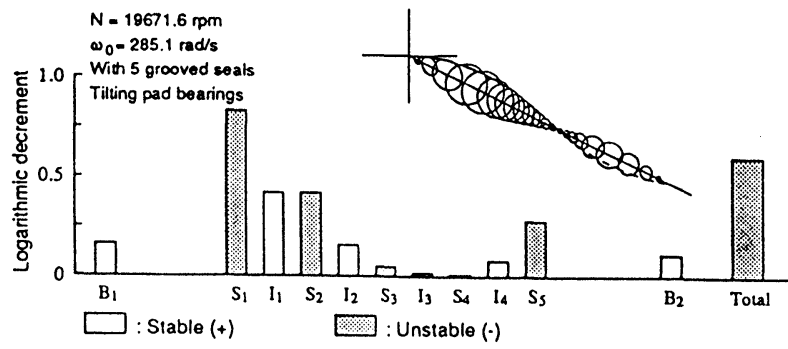

(d) With 2 tilting pad bearings and 5 grooved seals

FIGURE 8 Logarithmic decrement of multi-stage pump.

effect of the individual fluid element on rotor stability is an effective method for the purpose of the evaluation of rotor stability and dynamic design. 
2. Rotor stability can be improved by replacing the unstable or other elements. Such replacements usually cause a variation on the logarithmic decrements of the other elements.

3. The effect of preswirl velocity in seals on the stability is consistent with that of the individual research on seals.

\section{Nomenclature}

$\begin{array}{ll}C(\mathrm{~N} \cdot \mathrm{s} / \mathrm{m}) & \text { Damping coefficient } \\ F(\mathrm{~N}) & \text { Force } \\ K(\mathrm{~N} / \mathrm{m}) & \text { Stiffness coefficient } \\ M(\mathrm{~kg}) & \text { Inertia coefficient } \\ N(\mathrm{rpm}) & \text { Rotating speed of rotor } \\ n & \text { Number of fluid elements } \\ V_{t}(\mathrm{~m} / \mathrm{s}) & \text { Preswirl velocity in seal } \\ x, y, z & \text { Coordinates } \\ \alpha & \text { Real part of eigenvalue } \\ \delta & \text { Logarithmic decrement } \\ \lambda & \text { Eigenvalue } \\ \phi & \text { Rotationary coordinates } \\ \psi & \text { Eigenvector } \\ \omega & \text { Eigenfrequency or imaginary part of eigenvalue } \\ \omega_{0} & \text { Eigenfrequency of rotor system without fluid }\end{array}$

\section{Subscripts}

$\begin{array}{ll}B & \text { Bearing } \\ I & \text { Impeller } \\ S & \text { Seal }\end{array}$

\section{Superscripts}

\section{References}

Black, H. F., 1969. Effects of Hydraulic Forces in Annular Pressure Seals on the Vibrations of Centrifugal Pump Rotors, Trans. ASME J. Mech. Eng. Sci., Vol. 11, no. 2, pp. 206-213.

Childs, D. W., 1983. Finite-Length Solutions for Rotordynamic Coefficients of Turbulent Annular Seals, Trans. ASME. J. Lubr. Technol., Vol. 105, pp. 437-445.

Diewald, W., and Nordmann, R., 1987. Dynamic Analysis of Centrifugal Pump Rotors With Fluid-Mechanical Interactions, ASME. Vibrations Conf., pp. 571-580.

Iwatsubo, T., Sheng, B. C., and Matsumoto, T., 1989. An Experimental Study on the Static and Dynamic Characteristics of Pump Annular Seals (2nd Report, The Dynamic Characteristics for Small Concentric Whirling Motion), Trans. JSME Series C, (in Japanese), Vol. 55, no. 510, pp. 317-322.

Iwatsubo, T., and Sheng, B. C., 1989. Evaluation of Dynamic Characteristics of Parallel Grooved Seals by Theory and Experiment, Proc. of 1st Asia Vibration Conference, pp. 498-504.

Iwsatsubo, T., and Sheng, B. C., 1990. An Experimental Study on the Static and Dynamic Characteristics of Damper Seals, Proc. of IFToMM Conference, pp. 307-312.

JSME, 1984. Data Collection of Static and Dynamic Characteristics of Journal Bearings, Publishing Company of Japanese Industry (in Japanese).

Kanki, H., and Kwakami, T., 1984. Experimental Study on the Dynamic Characteristics of Pump Annular Seals, I. Mech. E., C297/84, pp. 159-166.

Kurohashi, M., Iwatsubo, T., Kawabata, N., and Fujikawa, T., 1982. Evaluation of Rotor Stability by Means of Logarithmic Decrements Derived from Energy for the Individual Elements, Trans. JSME (in Japanese), Vol. 48, no. 430, pp. 825-832.

Nordmann, R., Dietzen, F. J., and Janson, W., 1986. Rotordynamic Coefficients and Leakage Flow of Parallel Grooved Seals and Smooth Seals, NASA cp-2443, pp. 129-153.

Ohashi, H., and Shoji, H., 1987. Lateral Fluid Forces on Whirling Centrifugal Impeller (2nd Report: Experiment in Vaneless Diffuser), Trans. ASME. J. Fluids Eng., Vol. 109, pp. 100-106.

Yang, B. S., Iwatsubo, T., and Kawai, R., 1985. Effects of Seals on the Stability of Pump Rotor System, Trans. JSME (in Japanese), Vol. 51, no. 470 , pp. $2479-2486$ 

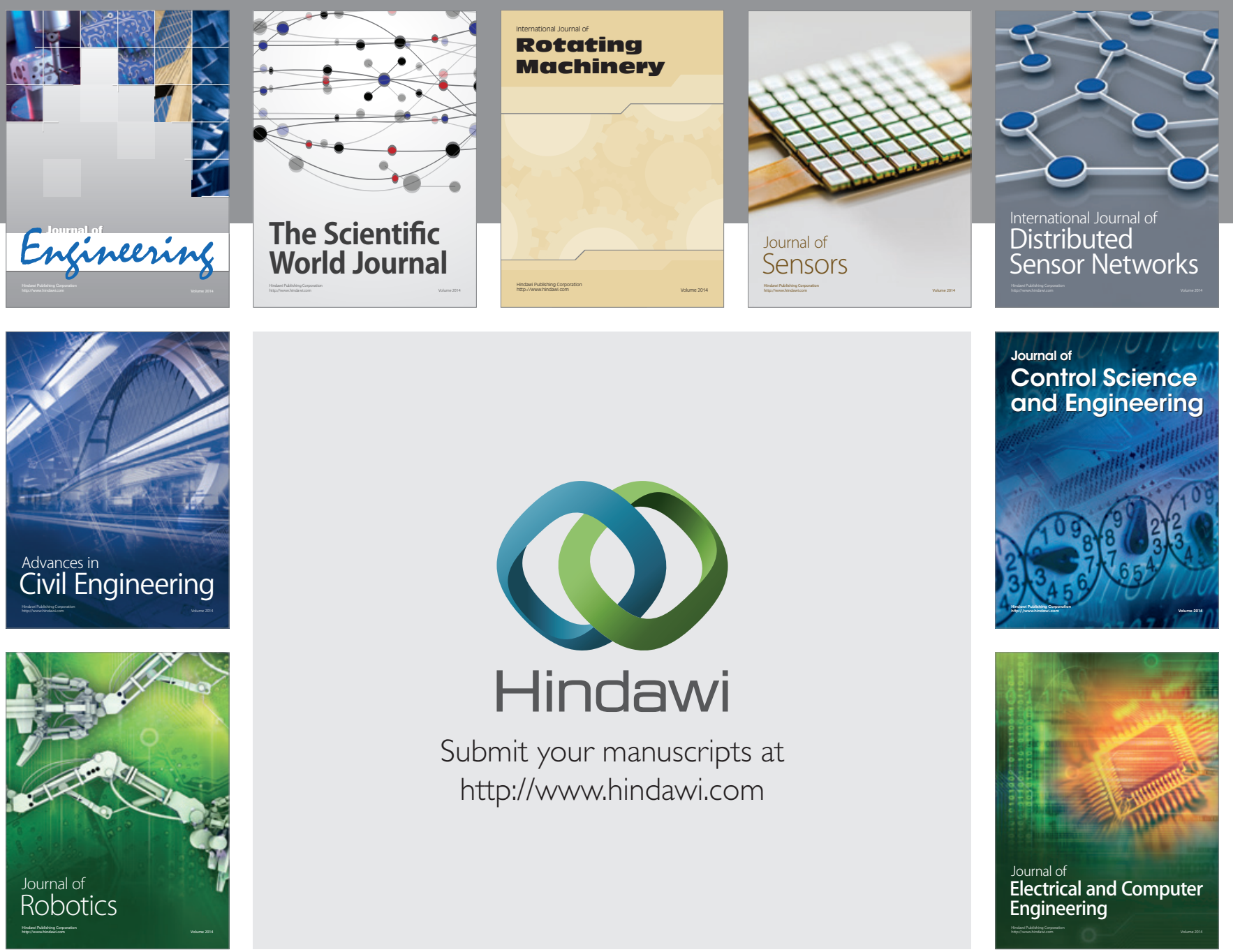

Submit your manuscripts at

http://www.hindawi.com
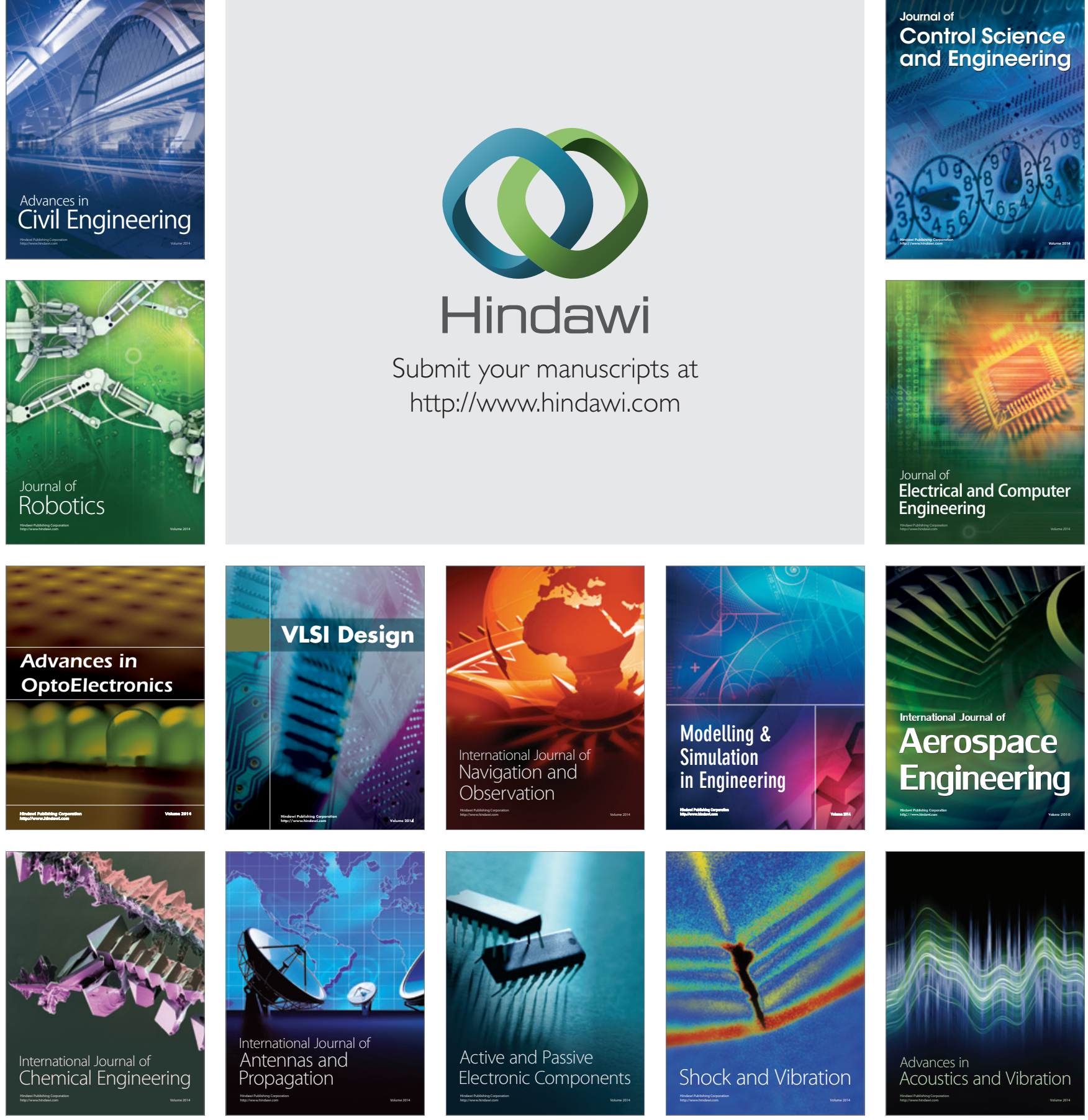